\section{BRST symmetry and quantum mechanics on homogeneous spaces}

To cite this article: B P Dolan 1990 J. Phys. A: Math. Gen. 234439

View the article online for updates and enhancements.

\section{Related content}

- On the relation between operator
$\frac{\text { constraint-, master constraint-, reduced }}{\text { phase space- and path integral }}$
$\frac{\text { quantisation }}{\text { Muxin Han and T Thiemann }}$
- Histories quantization of parameterized
Systems: I
K Savvidou and C Anastopoulos
- Topical Review
Abhay Ashtekar and Jerzy Lewandowski

\section{Recent citations}
Dirac and reduced quantization: A Lagrangian approach and application to coset spaces
C. R. Ordo?n?ez and J. M. Pons
Superselection rules from Dirac and BRST quantisation of constrained systems Landsman, N. P. and Linden, N.
- Dirac versus reduced quantization: a geometrical approach G Kunstatter




\title{
BRST symmetry and quantum mechanics on homogeneous spaces
}

\author{
B P Dolan \\ Department of Mathematical Physics, St Patrick's College, Maynooth, Ireland and School \\ of Theoretical Physics, Dublin Institute for Advanced Studies, 10 Burlington Road, Dublin \\ 4 , Ireland
}

Received 5 June 1989

\begin{abstract}
The quantum mechanical amplitudes for a free, non-relativistic particle moving on a finite-dimensional, homogeneous space $\mathscr{F} / \mathscr{R}$ are derived by applying first class constraints to a particle moving on the Lie group $\mathscr{S}$, where the amplitudes are already known exactly. This requires gauge fixing, and gauge invariance of the final result is ensured by invoking BRST symmetry and employing ghosts.
\end{abstract}

\section{Introduction}

Anti-commuting ghost fields were introduced into field theory in an attempt to construct a sensible quantum theory of the gravitational and non-Abelian Yang-Mills fields. They were found to be necessary in order to maintain unitarity once the gauge is fixed $[1,2]$. More recently it has been realized that they have a perfectly reasonable classical interpretation $[3,4]$ as 1 -forms on the group of all possible gauge transformations, hence their anti-commuting property [5]. Since gauge symmetries are a sign of first class constraints in phase space, [6], ghosts always play a role in systems with such constraints. This is true not only in field theories with an infinite number of degrees of freedom but also in finite-dimensional systems with first class constraints. Indeed, for classical systems, ghosts are even necessary in order to implement reparametrizations of the constraints as canonical transformations (in an extended phase space involving ghost degrees of freedom) $[3,7,8]$.

When quantizing systems with constraints, one has to decide whether to remove the unphysical degrees of freedom first and only quantize the true degrees of freedom or to quantize the unconstrained system first, including the unphysical degrees of freedom, and only project out the true physical degrees of freedom after quantization. Often these two procedures appear to lead to different results. In many situations quantization of the unconstrained system is easier and so this is the path of least resistance, but the only way to be sure that one has arrived at the correct quantum theory is to follow the former course and remove the unphysical degrees of freedom first. It was shown in $[7,8]$ that, at least for finite-dimensional systems with first class constraints linear in momenta, these two procedures can always be made to commute provided the measure on the Hilbert space of the unconstrained systems is chosen properly, its form being dictated by the constraints themselves. The physical Hilbert space is then identified with a subspace of the extended Hilbert space of the unconstrained system (extended to include ghosts for the constraints). 
The purpose of this paper is to point out there is a large class of examples for which the construction of $[7,8]$ can be applied, namely non-relativistic free particles moving on homogeneous spaces $\mathscr{M}=\mathscr{S} / \mathscr{R}$, where $\mathscr{S}$ is a semi-simple Lie group and $\mathscr{R} \subset \mathscr{S}$ a Lie subgroup. The quantum mechanics of particles moving on homogeneous spaces has already received much attention in the literature [9-13] but none of these approaches has emphasized the role of ghosts, which plays an important part in ensuring that quantizing first and then reducing commutes with reducing first and then quantizing. The basic strategy is to treat the particle moving on $\mathscr{S} / \mathscr{R}$ as a constrained system by applying constraints to a particle moving on $\mathscr{F}$, where quantum mechanical amplitudes can be calculated exactly [14-16]. It will be shown that the Cartan-Killing metric on $\mathscr{S}$ gives the correct measure for the Hilbert space $\mathscr{H}_{f f}$ of the unconstrained system for a particle moving on $\mathscr{H}$, as defined in [7]. $\mathscr{H}_{; f}$ is then extended to include ghosts for the constraints, giving an extended pseudo-Hilbert space $\tilde{\mathscr{H}}_{\mathscr{f}}$, and the physical Hilbert space of $\mathscr{I} / \mathscr{R}$, denoted by $\mathscr{H}_{\mathscr{H} / \mathscr{A}}$, is obtained by projecting out the physical subspace $\mathscr{H}_{f / \not \pi}=\mathscr{H}_{\text {Phys }} \subset \tilde{\mathscr{H}}_{f f}$ of $\tilde{\mathscr{H}}_{f t}$. This projection requires a gauge choice to remove the unphysical degrees of freedom, but the construction of $[7,8]$ ensures that the final result is independent of this choice. Thus the quantum amplitudes for a particle moving on $\mathscr{S} / \mathscr{R}$ can be obtained unambiguously from those of a particle moving on $\mathscr{Y}$, which are known exactly $[14,15]$.

As an illustration of the technique, we treat the example of a 2 -sphere, $\operatorname{SU}(2) / \mathrm{U}(1)$. This particular example is already well studied and the results presented here are not new, but the method of deriving them is different and it exhibits clearly the role of the ghosts.

In the second section, the relevant aspects of constrained, finite-dimensional Hamiltonian systems and classical BRST ghosts are reviewed. The exposition follows closely the treatment in [3]. The third section deals with the quantum mechanics of systems with first class constraints linear in momenta, as developed in $[7,8]$. In section 4 , these analyses are applied to a free, non-relativistic particle moving on $\mathscr{S} / \mathscr{R}$. Section 5 treats the explicit example of $S U(2) / U(1)$ using these techniques, and finally the results are summarized in section 6 .

\section{Classical constrained Hamiltonian systems}

Consider a free, non-relativistic particle moving on an $N$-dimensional curved manifold $\mathscr{Y}$, with coordinates $Q^{\alpha}, \alpha=1, \ldots, N$, and invertible metric $G_{\alpha \beta}(Q)$. The Lagrangian is

$$
L(Q, \dot{Q})=\frac{1}{2} G_{\alpha \beta}(Q) \dot{Q}^{\alpha} \dot{Q}^{\beta}
$$

The phase space of $\mathscr{S} \mathscr{P}=T^{*}(\mathscr{S})$, has a natural symplectic structure, and the Hamiltonian is (with coordinates $\left(Q^{\alpha}, P_{\alpha}\right)$ on $\mathscr{P}$ )

$$
H=\frac{1}{2} G^{\alpha \beta}(Q) P_{\alpha} P_{\beta}
$$

If we now apply $d$ constraint equations, $\Phi_{i}(Q, P)=0, i=1, \ldots, d$, to $\mathscr{P}$ we get a $(2 N-d)$-dimensional manifold $\mathscr{C}$. (To avoid unnecessary complications, it is assumed that the constraints are irreducible.) If $\mathscr{C}$ is itself a symplectic manifold, with symplectic structure inherited from $\mathscr{S}$, then the constraints $\Phi_{i}$ are called second class. If $\mathscr{C}$ is co-isotropic (this means that there exists a canonical transformation to a coordinate system $\left(Q^{\alpha}, P_{\alpha}\right)$ on $\mathscr{P}$ such that $\mathscr{C}$ has the same number of coordinates $Q$ as $\mathscr{P}$ but 
only $N-d$ coordinates $P$, or vice versa) then the constraints are called first class. The standard test for first class constraints is to compute all their Poisson brackets among themselves, $\frac{1}{2} d(d-1)$ of them. If these can all be written as linear combinations of the original constraints, $\Phi_{i}(Q, P)$, then they are first class. In other words

$$
\left\{\Phi_{i}, \Phi_{j}\right\}=C(Q, P)_{i j}{ }^{k} \Phi_{k}
$$

where the 'structure functions' $C(Q, P)_{i}{ }^{k}$ are, in general, functions of $Q^{\text {cr }}$ and $P_{\alpha}(\{\}$, here denotes the Poisson bracket in $\mathscr{P}$ ). For a general constrained system the constraints will be a mixture of both first and second class types, but in this paper, only first class constraints will be considered.

The constrained manifold $\mathscr{C}$ can be further constrained to the reduced phase space in the following way [3]. Let $F(Q, P)$ be a differentiable function on $\mathscr{P}$. Then define $d$ vector fields $\boldsymbol{\Phi}_{i}$ on $\mathscr{P}$ by

$$
\Phi_{i} F=\left\{\Phi_{i}, F\right\} \text {. }
$$

The algebra of these $d$ vector fields, obtained by taking Lie derivatives, does not close in general since

$$
\left[\boldsymbol{\Phi}_{i}, \boldsymbol{\Phi}_{j}\right] F \equiv \boldsymbol{\Phi}_{i} \boldsymbol{\Phi}_{j} F-\boldsymbol{\Phi}_{j} \boldsymbol{\Phi}_{i} F=C_{i j}{ }^{k}(Q, P) \boldsymbol{\Phi}_{k} F+\Phi_{k}\left\{C_{1\}}{ }^{k}(Q, P), F\right\} .
$$

However their algebra does close on the constrained manifold $\mathscr{C}$, where $\Phi_{k}$ vanish. If the $C_{i j}{ }^{k}(Q, P)$ are actually constants independent of $Q^{\alpha}$ and $P_{\alpha}$, then the algebra of $\boldsymbol{\Phi}_{i}$ closes everywhere in $\mathscr{P}$ and they are generators of a $d$-dimensional Lie group, $\mathscr{R}$. For our purposes, only constant $C_{i j}{ }^{k}$ need be considered, so this will be assumed from now on. The constraints then generate an action of the group $\Re$ on $\mathscr{C}$. Factoring out by this action gives the reduced phase space, the true phase space of the constrained system, see figure 1 .

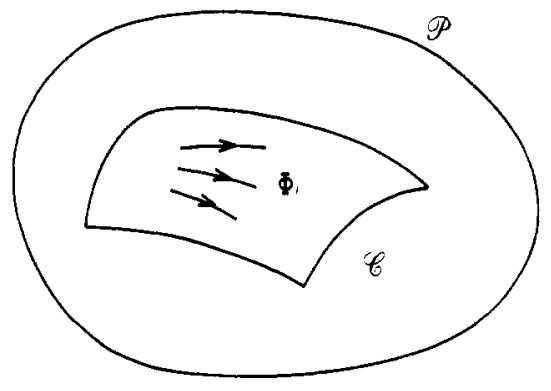

Figure 1. Factoring out the action of the group $\not R$ on $G$ in order to obtain the true phase space of the constrained system.

An explicit representation of the reduced phase space can be obtained by choosing a gauge. This requires $d$ functions $\chi^{\prime}(Q, P)$ on $\mathscr{P}$ whose Poisson brackets with $\Phi_{i}$ are non-vanishing on $\mathscr{C}$. The submanifold of $\mathscr{C}$ on which $\chi^{\prime}$ vanish is a symplectic manifold which is the phase space of the constrained system (provided the gauge conditions can be globally well defined and there are no ambiguities of the Gribov type [17]). In general this will be the phase space for a free particle moving on a manifold $M$ of dimension $n=N-d$ which can be embedded in $\mathscr{T}$. Denote a local coordinate system on $\mathcal{M}$ by $q^{\mu}, \mu=1, \ldots, n$, with Riemannian metric $g_{\mu \nu}$ which is the pull back, under the embedding, of $G_{\alpha \beta}$. The dynamics of this system could equally well have been obtained directly by considering the Lagrangian

$$
\mathscr{L}(q, \dot{q})=\frac{1}{2} g_{\mu \nu}(q) \dot{q}^{\mu} \dot{q}^{\prime \prime}
$$


leading to the Hamiltonian

$$
h(q, p)=\frac{1}{2} g^{\mu \nu}(q) p_{\mu} p_{\nu}
$$

where $\left(q^{\mu}, p_{\mu}\right)$ are coordinates on the $2 n$-dimensional phase space, $T^{*}(\mathscr{M})$.

Of course any linear combination

$$
\Phi_{i^{\prime}}=\Lambda_{i^{\prime}}{ }^{i}(Q, P) \Phi_{i}
$$

is also a set of constraints which picks out the same submanifold $\mathscr{C}$ of $\mathscr{P}$ as $\Phi_{i}$, provided det $\Lambda \neq 0$. It is in general not possible to implement the transformation (8) as a canonical transformation on $\mathscr{P}$. However, as demonstrated in [3], if the phase space $\mathscr{P}$ is extended to include ghosts, it is possible to implement (8) as a canonical transformation in the extended phase space. Introduce a classical anti-commuting ghost coordinate $\eta^{i}$ for each constraint $\Phi_{\text {. }}$. Then extend the configuration space $\mathscr{S}$ to $\tilde{\mathscr{S}}$ by including the ghosts. Local coordinates on $\tilde{\mathscr{S}}$ will be denoted by $\left(Q^{\alpha}, \eta^{i}\right)$. Momenta conjugate to the ghosts are represented by $\rho_{i}$ with graded Poisson bracket

$$
\left\{\eta^{\prime}, \rho_{j}\right\}=\delta^{i}
$$

and phase space is extended to $\tilde{\mathscr{P}}$ with coordinates $\left(Q^{\alpha}, \eta^{\prime}, P_{\alpha}, \rho_{i}\right)$. $\tilde{\mathscr{P}}$ has a graded structure in that any element can be uniquely split into terms with definite ghost number, where the $\eta^{i}$ have ghost number 1 and the $\rho_{i}$ have ghost number -1 . The transformations (8) can be realized as canonical transformations on $\tilde{\mathscr{P}}$.

Physical observables of the constrained system are represented as functions $F(Q, P)$ on $\mathscr{P}$ with the special property that

$$
\left\{F, \Phi_{i}\right\}=F_{i}{ }^{j} \Phi_{j}
$$

for some functions $F_{1}{ }^{j}$, i.e. the Poisson bracket of $F$ with the constraints vanishes on the constrained manifold $\mathscr{C} . F$ is then gauge invariant on $\mathscr{C}$ and $\Phi_{i}$ generate gauge transformations. Any such observable is invariant on $\mathscr{C}$ under the change

$$
F \rightarrow F+F^{i} \Phi_{i}
$$

for arbitrary $F^{i}$.

For any set of first class constraints, one can construct a function with ghost number 1 on $\tilde{P}$, called the BRST operator:

$\Omega=\eta^{i} \Phi_{i}+\eta^{i} \eta^{j} \rho_{k} C_{i j}{ }^{k}(Q, P)+$ terms which vanish when $C_{i j}{ }^{k}$ are constants

which has the property that its graded Poisson bracket with itself vanishes

$$
\{\Omega, \Omega\}=0 \text {. }
$$

Any gauge invariant observable $F(Q, P)$ on $\mathscr{P}$ can be extended to a ghost number 0 function $\tilde{F}$ on $\tilde{P}$, called a BRST extension of $F$, for which

$$
\{\tilde{F}, \Omega\}=0 \text {. }
$$

Equation (14) does not determine $\tilde{F}$ uniquely, but only up to a term of the form $\{\Omega, W\}$ where $W$ is any ghost number -1 function, i.e. if $\tilde{F}_{1}$ is one BRsT extension of $F$ then

$$
\tilde{F}_{2}=\tilde{F}_{1}+\{\Omega, W\}
$$


is another, which also satisfies (14) (since $\{\Omega,\{\Omega, W\}\}=0$ by the Jacobi identity and nilpotency of $\Omega(13)$ ). Thus, gauge-invariant functions on $\mathscr{b}$ are in one-to-one correspondence with the elements of the ghost number 0 cohomology class of $\Omega$ on $\tilde{\mathscr{P}}$. Explicitly, a BRST extension of $F$ is given by [4]

$$
\tilde{F}=F+\eta^{\prime} F_{i}^{j} \rho_{j}+\text { higher-order ghost terms. }
$$

\section{Quantization of constrained systems}

The aim is to quantize the particle moving on $\mathcal{M}$. As mentioned in the introduction, there are two ways of doing this.

(i) Reduce first and then quantize, i.e. work directly with $\mathscr{M}$ or $T^{*}(\mathcal{M})$, introduce a Hilbert space $\mathscr{H}_{\| \mu}$ and quantize $h(q, p)$ on $\mathscr{H}_{. \mu}$.

(ii) Quantize first and then reduce, i.e. introduce a Hilbert space $\mathscr{H}_{i f}$, quantize $H(Q, P)$ on $\mathscr{H}_{f f}$ and then try to project down from $\mathscr{H}_{f f}$ to $\mathscr{H}_{t 1}$.

In many circumstances, it is much easier to quantize a particle on $\mathscr{S}$ than one on $\mathcal{M}$ in which case route (ii) is the best way to proceed. However, one often runs into difficulties with these two procedures and can end up with different quantum mechanics, depending on which route is adopted. It is shown in $[7,8]$ that, at least when the reduction can be implemented via first class constraints linear in momenta, these two routes can be made to commute by introducing ghosts, extending $\mathscr{H}_{5 f}$ to a (pseudo)Hilbert space, $\tilde{\mathscr{H}}_{f}$, and then projecting out the physical Hilbert space $\mathscr{H}_{.4}=\mathscr{H}_{\text {Phys }} \subset \tilde{\mathscr{H}}_{4}$ from $\tilde{\mathscr{H}}_{\mathscr{f}}$. It is emphasized in [7] that this process is only consistent if the Hilbert space metric on $\mathscr{H}_{f f}$ is the pull-back of that on $\mathscr{H}_{11}$ under the projection from $\mathscr{H}_{f f}$ to the physical Hilbert space. The rest of this section is devoted to an explanation of the construction of the extended (pseudo)-Hilbert space and its physical subspace, as described in $[7,8]$.

One problem that we have to face is that of factor ordering in the Hamiltonians. We shall adopt one which is adapted to the Schrödinger representation.

Take the quantum Hamiltonian, $\hat{h}(q, p)$, on $\mathscr{H}_{\text {il }}$ to be

$$
\hat{h}=\frac{1}{2} \hat{g}^{-1 / 4} \hat{p}_{\mu} \hat{g}^{1 / 2} \hat{g}^{\mu \nu} \hat{p}_{\nu} \hat{g}^{-1 / 4} \quad \text { with } \quad g=\operatorname{det} g_{\mu \nu}
$$

In the Schrödinger representation this is

$$
\hat{h}=-\frac{1}{2 \sqrt{g}} \partial_{\mu}\left(\sqrt{g} g^{\mu \nu} \hat{o}_{\nu}\right)=-\frac{1}{2} \nabla
$$

where

$$
\hat{p}_{\mu}=-\mathrm{i}\left(\partial_{\mu}+\frac{1}{2} \partial_{\mu} \ln \sqrt{g}\right)
$$

and $\nabla$ is the Laplacian on $\mathcal{M}$.

We can represent the Hilbert space $\mathscr{H}_{\mathfrak{H}}$ in the coordinate basis $|q\rangle$ and use $g_{\mu \nu}(q)$ as the Hilbert space metric. Thus

$$
\left\langle q^{\prime} \mid q\right\rangle=\frac{1}{\sqrt{g}} \delta^{(n)}\left(q^{\prime}-q\right) .
$$

The states are then

$$
|\psi\rangle=\int \psi(q)|q\rangle d \mu
$$


where $\psi(q)$ is a square integrable function on $\mathcal{M}$, and $\mathrm{d} \mu_{: t 1}=\sqrt{\mathrm{g}} \mathrm{d}^{n} q$ is the measure on $\mathscr{H}_{u}$ (with this measure $\hat{p}_{\mu}$, as defined in (19), is Hermitian). The Hermitian inner product between two states is

$$
\left\langle\psi_{1} \mid \psi_{2}\right\rangle=\int \psi_{1}^{*}(q) \psi_{2}(q) \mathrm{d} \mu_{t t} .
$$

In the particular instances when the constraints are linear in momenta, $\mathscr{H}_{\text {.1 }}$ can also be derived from $\mathscr{H}_{f}$ as follows. Let $|Q\rangle$ be the coordinate basis for $\mathscr{H}_{i f}$ and introduce a measure, $\mathrm{d} \mu_{\text {ff }}$, on $\mathscr{S}$ defined as in [7]. With $\Phi_{i}=\Phi_{i}{ }^{\alpha}(Q) P_{\alpha}$, define

$$
\Phi_{\alpha_{1} \ldots \alpha_{n}}=\varepsilon_{\alpha_{1} \ldots \alpha_{n} \beta_{1} \ldots \beta_{d}} \Phi_{1}^{\beta_{1}} \ldots \Phi_{d}^{\beta_{d}}
$$

where $\varepsilon_{\alpha_{1} \ldots \alpha}$ is the totally antisymmetric density with $\varepsilon_{1 \ldots N}=1$ and

$$
\|\Phi\|^{-2}=\frac{1}{n !} \Phi_{\alpha_{1} \ldots \alpha_{n}} G^{\alpha_{1} \beta_{1}} \ldots G^{\alpha_{n} \beta_{n}} \Phi_{\beta_{1} \ldots \beta_{n}} .
$$

Then

$$
\mathrm{d} \mu_{\mathscr{f}}=\|\Phi\| \mathrm{d}^{n} Q
$$

gives the required measure on $\mathscr{H}_{f t}$ and its extension $\tilde{\mathscr{H}}_{f}$. It is emphasized in [7] that it is not actually necessary that $G^{\alpha \beta}$ have an inverse in order to define the measure $\mathrm{d} \mu_{f f}$. In particular, if $\|\Phi\| \neq \sqrt{G}$ then procedures (i) and (ii) above do not commute, and indeed many examples in the literature of this non-commutativity are traceable to this inequality.

Having defined the measure on $\mathscr{H}_{f,}$, the basis states are now normalized to

$$
\left\langle Q^{\prime} \mid Q\right\rangle=\frac{1}{\|\Phi\|} \delta^{(N)}\left(Q^{\prime}-Q\right)
$$

The states are then

$$
|\Psi\rangle=\int \Psi(Q)|Q\rangle \mathrm{d} \mu_{: f}
$$

where $\Psi(Q)$ is a square integrable function on $\mathscr{S}$. The Hermitian inner product between two states is

$$
\left\langle\Psi_{1} \mid \Psi_{2}\right\rangle=\int \Psi_{1}^{*}(Q) \Psi_{2}(Q) \mathrm{d} \mu_{\mathscr{s}} .
$$

The extended pseudo-Hilbert space $\tilde{\mathscr{H}}_{f}$ has coordinate basis $\left|Q ; i_{1}, \ldots, i_{p}\right\rangle$ where $p=1, \ldots, d$, and inner product [8]

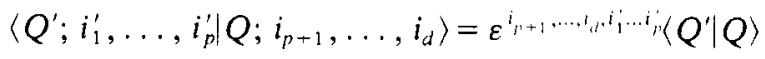

all others vanishing. $\tilde{\mathscr{H}}_{f,}$ has $N$ commuting coordinates and $d$ anti-commuting coordinates. Note that $\left\langle Q^{\prime} ; \mid Q ;\right\rangle=0$, where $|Q ;\rangle$ is a ghost number 0 state. The ghost number $p$ basis states, $\left|Q ; i_{1}, \ldots, i_{p}\right\rangle$, can be thought of as being obtained from $|Q ;\rangle$ using Hermitian ghost creation operators, $\hat{\eta}=\hat{\eta}^{+}$

$$
\left|Q ; i_{1}, \ldots, i_{p}\right\rangle=\hat{\eta}_{i_{1}} \ldots \hat{\eta}_{t_{p}}|Q ;\rangle
$$

The states in $\tilde{\mathscr{H}}_{i f}$ can thus be written as

$$
|\tilde{\Psi}\rangle=\sum_{p=0}^{d} \frac{1}{p !} \int \Psi_{i_{1} \ldots i_{p}}(Q)\left|Q ; i_{1}, \ldots, i_{p}\right\rangle \mathrm{d} \mu_{i,} .
$$


We are using here a Schrödinger representation for the ghosts, where the conjugate momenta would be anti-Hermitian $\hat{\rho}=-\hat{\rho}^{\dagger}$ represented by

$$
\hat{\rho}=-\mathrm{i} \frac{\delta}{\delta \eta}
$$

Strictly speaking, $\tilde{\mathscr{H}}_{s f}$ is a pseudo-Hilbert space, not a true Hilbert space, because the metric is not positive definite, the ghost sector has invariance $U\left(2^{d-1}, 2^{d-1}\right)$ rather than $\mathrm{U}\left(2^{d}\right)$.

To project out the physical Hilbert space of the constrained system it is necessary to choose a gauge, though the final result is independent of this choice. Let $\chi^{i}(Q, P)$ be a gauge choice. Then $\mathscr{H}_{\text {Phys }} \subset \tilde{\mathscr{H}}_{\text {If }}$ is the space of states of the form

$|\Psi\rangle_{\text {Phys }}=\frac{1}{\sqrt{2}} \int \Psi(Q)\left(|Q ;\rangle+\mathrm{i}^{3 d(d-1) / 2} \delta\left(\chi^{1}\right) \ldots \delta\left(\chi^{d}\right)|\chi, \Phi||Q ; 1, \ldots, d\rangle\right) \mathrm{d} \mu_{\text {; }}$

which, in addition, are annihilated by $\hat{\Omega}$, i.e. $\{\Omega, \Psi(Q)\}=0$. Here $|\chi, \Phi|$ is the modulus of the determinant of the $d \times d$ matrix of Poisson brackets $\left\{\chi^{\prime}, \Phi_{j}\right\}$. It is shown in [8] that these states are BRST invariant. The physical Hilbert space defined here does not have ghost number 0 . To be more precise, one should introduce anti-ghosts $\bar{\eta}_{i}$ and conjugate momenta $\bar{\rho}^{-i}$ and further extend the pseudo-Hilbert space to a space with basis

$$
\left|Q ; i_{1}, \ldots, i_{p} ; j_{1}, \ldots, j_{p^{\prime}}\right\rangle=\hat{\eta}^{i_{1}} \ldots \hat{\eta}^{i} \hat{\bar{\eta}}_{j_{1}} \ldots \hat{\bar{\eta}}_{j_{p}}|Q ; ;\rangle
$$

which have ghost number $p-p^{\prime}$. The ghost-anti-ghost sector has invariance $\mathrm{U}\left(2^{2 d-1}, 2^{2 d-1}\right)$. Then the physical states are constructed as

$|\Psi\rangle_{\text {Phys }}=\frac{1}{\sqrt{2}} \int \Psi(Q)\left(|Q ; ;\rangle+\mathrm{i}^{3 d(d-1)} \delta\left(\chi^{1}\right) \ldots \delta\left(\chi^{d}\right)|\chi, \Phi \| Q ; 1, \ldots, d ; 1 \ldots, d\rangle\right) \mathrm{d} \mu_{; f}$

with $\{\Omega, \Psi(Q)\}=0$, which do have ghost number 0 . The anti-ghosts are an unnecessary complication for our purposes and shall be ignored in what follows.

As noted above, it is not necessary that $G^{\alpha \beta}$ have an inverse in order to define the measure on $\mathscr{S}$. However, when it does have an inverse we can express the dynamics on $\tilde{\mathscr{H}}_{S g}$ as follows. The Hamiltonian operator, $\hat{H}$, on $\tilde{\mathscr{H}}_{y t}$ is obtained from the BRST extension (16) of the Hamiltonian, $\hat{H}$, on $\mathscr{H}_{\text {sf }}$. For the latter the same factor ordering is adopted as for $\hat{h}$,

$$
\hat{H}=\frac{1}{2} \hat{G}^{-1 / 4} \hat{P}_{\alpha} \hat{G}^{1 / 2} \hat{G}^{\alpha \beta} \hat{P}_{\beta} \hat{G}^{-1 / 4}
$$

where $G=\operatorname{det} G_{\alpha \beta}$. As before, in the Schrödinger representation with

$$
\hat{P}_{\alpha}=-\mathrm{i}\left(\partial_{\alpha}+\frac{1}{2} \partial_{\alpha} \ln \sqrt{G}\right)
$$

this is

$$
\hat{H}=-\frac{1}{2 \sqrt{G}} \partial_{\alpha}\left(\sqrt{G} G^{\alpha \beta} \partial_{\beta}\right)=-\frac{1}{2} \square
$$

where $\square$ is the Laplacian on $\mathscr{F}$.

For the Hamiltonian $\hat{\hat{H}}$, on $\tilde{\mathscr{H}}_{f}$, use the BRST extension of (38) (for details of factor ordering, which will not be relevant here, see [8]). 


\section{Quantum mechanics on coset spaces}

Our intention is to apply the formalism of the previous section to the problem of a particle moving on the space of cosets of a (compact) $N$-dimensional semi-simple Lie group $\mathscr{S}$ with respect to the left action of a subgroup $\Re$ (of dimension $d$ ). The left action of $\mathscr{R}$ on $\mathscr{S}$ induces a fibration of $\mathscr{S}$ leading to the fibre bundle structure

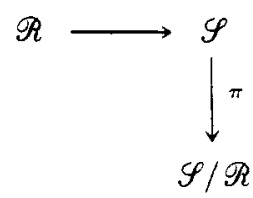

with total space $\mathscr{S}$, fibre $\mathscr{R}$, base space $\mathscr{S} / \mathscr{R}$ and projection $\pi . \mathscr{S} / \mathscr{R}$ is the configuration space of the physical system which was called $\mathcal{M}$ in the more general situation considered in the previous sections.

Let $G$ be the Cartan-Killing metric on $\mathscr{S}$ and $g$ the $\mathscr{S}$ invariant metric on $\mathscr{S} \mathscr{R}$ obtained by pushing $G^{-1}$ forward by the projection $\pi$, i.e. given $G$, define $g$ by

$$
\pi_{*}\left(G^{-1}\right)=g^{-1}
$$

where $G^{-1}$ and $g^{-1}$ are rank-2 contravariant tensors on $\mathscr{S}$ and $\mathscr{S} \mathscr{R}$, respectively. Then the pull-back of $g$ to $\mathscr{S}$ is $\mathscr{S}$ invariant, since $g$ is. Since $G$ is the unique $\mathscr{S}$ invariant metric on $\mathscr{S}$ (up to a constant normalization), we have

$$
\pi^{*}(g)=G
$$

Hence, in the quantum theory, the Hilbert space metric on $\mathscr{H}_{\mathscr{S}}$ is the pull-back of that on $\mathscr{H}_{\mathscr{S} / \mathscr{A}}$ and the construction of $[7,8]$ can be applied, with $\|\Phi\|=\sqrt{G}$.

Let $K_{A}=K_{A}{ }^{\alpha}(Q) \partial_{\alpha}$ be Killing vectors on $\mathscr{S}$ which generate the Lie algebra of $\mathscr{S}$, $A=1, \ldots, N$,

$$
\left[\boldsymbol{K}_{A}, \boldsymbol{K}_{B}\right]=C_{A B}{ }^{C} \boldsymbol{K}_{C} .
$$

On a semi-simple Lie group the Killing vectors can always be chosen to be orthonormal so that in a coordinate basis the (inverse) Cartan-Killing metric is

$$
G^{-1}=\delta^{A B} K_{A} K_{B}
$$

or in components

$$
G^{\alpha \beta}=K_{A}^{\alpha}(Q) K_{B}{ }^{\beta}(Q) \delta^{A B} \text {. }
$$

The $\mathscr{S}$ invariant metric $g$ on $\mathscr{R}$ can be obtained as follows [18]. Denote the elements of $\mathscr{S}$ by $s$, then $s^{-1} \mathrm{~d} s$, where $\mathrm{d}$ is the exterior derivative on $\mathscr{S}$, is a Lie algebra valued 1 -form in $T^{*}(\mathscr{S})$ and can thus be decomposed as

$$
s^{-1} \mathrm{~d} s=e^{A} T_{A}=e^{i} T_{i}+e^{m} T_{m}
$$

where $T_{A}$ are the generators of the Lie algebra of $\mathscr{S}, T_{i}$ are the generators of the Lie algebra of $\mathscr{R}(i=1, \ldots, d)$ and $T_{m}$ span the complement of the Lie algebra of $\mathscr{R}$ in the Lie algebra of $\mathscr{S}(m=d+1, \ldots, N)$. Then $e^{A}$ are orthonormal 1 -forms for $G$

$$
G=e^{A} e^{B} \delta_{A B}
$$

and $e^{m}$ are orthonormal 1 -forms for $g$

$$
g=e^{m} e^{n} \delta_{m n} .
$$


This means that the measure on $\mathscr{S}$ decomposes into the product of the $\mathscr{S}$-invariant measure on $\mathscr{S} / \mathscr{R}$ and the measure on $\mathscr{R}$. To see this, choose coordinates $\left(Q^{\alpha}\right)=$ $\left(Q^{\bar{\alpha}}, q^{\mu}\right)$ on $\mathscr{S}$, where $\bar{\alpha}=1, \ldots, d$ are coordinates on $\mathscr{R}$. Then

$$
e^{i}=e_{\alpha}^{i} \mathrm{~d} Q^{\tilde{\alpha}}+e^{i}{ }_{\mu} \mathrm{d} q^{\mu} \quad e^{m}=e^{m}{ }_{\mu} \mathrm{d} q^{\mu}
$$

(this is always possible, since $e^{m}$ are orthonormal 1-forms for $\mathscr{R}$ ). Thus, as a matrix,

$$
e_{\alpha}^{A}=\left(\begin{array}{cc}
e_{\bar{\alpha}}^{i} & e^{i}{ }_{\mu} \\
0 & e^{m}{ }_{\mu}
\end{array}\right)
$$

so

$$
\sqrt{\bar{G}}=\operatorname{det}\left(e^{A}{ }_{\alpha}\right)=\operatorname{det}\left(e_{\bar{\alpha}}^{i}\right) \operatorname{det}\left(e^{m}{ }_{\mu}\right)=\sqrt{g \bar{G}}
$$

where $\bar{G}=\operatorname{det} \bar{G}_{\bar{\alpha} \bar{\beta}}$, with $G_{\bar{\alpha} \bar{\beta}}$ the Cartan-Killing metric on $\mathscr{R}$. In other words

$$
\mathrm{d} \mu_{\mathscr{S}}=\mathrm{d} \mu_{\Re} \mathrm{d} \mu_{\mathscr{S} / \Re} \text {. }
$$

Of course $e^{A}{ }_{\alpha}$ are related to the Killing vector components $K_{A}{ }^{\alpha}$ by

$$
\delta_{A B} e^{B}{ }_{\alpha}=G_{\alpha \beta} K_{A}{ }^{\beta} \text {. }
$$

Thus the Hilbert space measure on $\mathscr{H}_{\mathscr{Y}}$ simply decomposes as a product as in (51).

Now the quantum Hamiltonian operator on $\mathscr{H}_{4}$ is, using (38),

$$
\begin{aligned}
\hat{H} & =-\frac{1}{2 \sqrt{G}} \partial_{\alpha}\left(\sqrt{G} G^{\alpha \beta} \partial_{\beta}\right) \\
& =-\frac{1}{2 \sqrt{G}} \delta^{A B} \partial_{\alpha}\left(\sqrt{G} K_{A}{ }^{\alpha}(Q) K_{B}{ }^{\beta}(Q) \partial_{\beta}\right) \\
& =-\frac{1}{2} K_{A} K_{B} \delta^{A B} .
\end{aligned}
$$

Here we have used the fact that $K_{A}{ }^{\alpha}(Q)_{; \alpha}=0$ and $K_{A}{ }^{\alpha}(Q) \partial_{\alpha} \sqrt{G}=0$, since $K_{A}$ are Killing, leading to

$$
K_{A}{ }^{\alpha}(Q)_{; \alpha}=\frac{1}{\sqrt{G}} \partial_{\alpha}\left(K_{A}{ }^{\alpha}(Q) \sqrt{G}\right)=\partial_{\alpha} K_{A}{ }^{\alpha}(Q)=0 .
$$

Note that we also have

$$
K_{A}{ }^{\alpha}(Q) \hat{P}_{\alpha}=-\mathrm{i} K_{A}{ }^{\alpha}(Q)\left(\partial_{\alpha}+\frac{1}{2} \partial_{\alpha} \ln \sqrt{G}\right)=-\mathrm{i} K_{A} .
$$

Let the $d$ vector fields $K_{i}$ on $\mathscr{G}$ generate the subgroup $\mathscr{R}$. Classically we can obtain the reduced phase space for a particle moving on $\mathscr{S} / \mathscr{R}$ by first applying the constraints $\Phi_{i}=K_{i}^{\alpha}(Q) P_{\alpha}$, linear in momenta, to obtain the constrained space $\mathscr{C}$ and then factoring out by the orbits of the action, under Poisson bracket operation, of the constraints on $\mathscr{C}$ itself. The constraints are automatically first class, with structure constants, because $\mathscr{R}$ is a Lie group

$$
\left\{\Phi_{i}, \Phi_{j}\right\}=C_{i j}{ }^{k} \Phi_{k} .
$$

In the quantum theory, the constraints become operators on $\mathscr{H}_{s f}$

$$
\hat{\Phi}_{i}=-\mathrm{i} K_{i}=-\mathrm{i} K_{i}^{\alpha}(Q) \partial_{\alpha} .
$$


Being Killing vectors, these constraints all commute, using Poisson brackets, with the Hamiltonian $\hat{H},(38)$, hence we can take the BRST extension of $\hat{H}(16)$ to be trivial:

$$
\hat{\tilde{H}}=-\frac{1}{2} K_{A} K_{B} \delta^{A B}
$$

independent of the ghost fields.

Note that since we have chosen $K_{1}, \ldots, K_{d}$ to be orthonormal, it is guaranteed that the measure $\|K\| \mathrm{d}^{N} Q$, obtained by the procedure outlined in the previous section, (25), will be the same as the naive one $\sqrt{G} \mathrm{~d}^{N} Q$.

A coordinate basis for the space of physical states within $\tilde{\mathscr{H}}_{\mathscr{S}}$ can be obtained as follows. Choose a cross section, $\chi: \mathscr{S} / \mathscr{R} \rightarrow \mathscr{S}$, of the above fibre bundle. Thus for every point $c \in \mathscr{S} / \mathscr{R}$ choose (in a smooth fashion) an element $\chi(c)=s \in \mathscr{S}$ in the coset of $\mathscr{S}$ represented by $c$. This is equivalent to choosing a gauge and can be represented more concretely by $d$ gauge functions $\chi^{i}\left(Q^{\alpha}\right)$, which are independent of the momenta $P_{\alpha}$. Then the state

$$
|q ;\rangle_{\chi} \stackrel{\text { def }}{=} \frac{1}{\sqrt{2}} \int_{\mathscr{R}}\left\{|Q(s r) ;\rangle+\mathrm{i}^{3 d(d-1) / 2} \delta^{(d)}\left(\chi^{i}\right)|\chi, K \| Q(s r) ; 1, \ldots, k\rangle\right\} \mathrm{d} \mu_{\text {.R }}
$$

is annihilated by $\hat{\Omega}$. The integral here is over all $r \in \mathscr{R}$ while $q$ represents the coordinates of the point $c \in \mathscr{S} / \mathscr{R}$ and the semi-colon is meant to remind us that $|q ;\rangle_{x}$ is a state in the extended Hilbert space $\tilde{\mathscr{H}}_{f f}$. The state $|q ;\rangle_{x}$ is automatically independent of the choice of cross section, by the results of $[7,8]$, and is normalized so that

$$
{ }_{\chi}\left\langle q^{\prime} ; \mid q ;\right\rangle_{\chi}=\frac{1}{\sqrt{g}} \delta^{(n)}\left(q-q^{\prime}\right) .
$$

Any physical state can be written as a linear combination of (59).

The time evolution of $|q\rangle$ can now be obtained from the time evolution of $|q ;\rangle_{x}$ which is much easier to calculate. In particular

$$
\left\langle q^{\prime}|\hat{h}| q\right\rangle={ }_{x}\left\langle q^{\prime} ;|\hat{\tilde{H}}| q ;\right\rangle_{x}
$$

where $\left\langle q^{\prime}|\hat{h}| q\right\rangle$ are the matrix elements of the Hamiltonian in $\mathscr{H}_{\mathscr{S} / \mathscr{A}}$. The quantum mechanical amplitude for a particle moving on $\mathscr{S} / \mathscr{R}$ can now be written in terms of $\left\langle q^{\prime}|\hat{h}| q\right\rangle$, but is more easily evaluated using ${ }_{x}\left\langle q^{\prime} ;|\hat{H}| q ;\right\rangle_{x}$.

Consider the amplitude, $\left\langle q^{\prime}, t^{\prime} \mid q, t\right\rangle$, for a particle to go from the state $\left|q^{\prime}\right\rangle$ at time $t^{\prime}$ to the state $|q\rangle$ at time $t$ :

$$
\begin{aligned}
& \left\langle q^{\prime}, t \mid q, t\right\rangle={ }_{\chi}\left\langle q^{\prime} ; t^{\prime} \mid q ; t\right\rangle_{\chi} \\
& ={ }_{x}\left\langle q^{\prime} ;\left|\exp \left[\mathrm{i} \hat{\hat{H}}\left(t^{\prime}-t\right)\right]\right| q ;\right\rangle_{\chi} \\
& =\frac{1}{2} \iint_{\mathscr{A}}\left\{\left\langle Q^{\prime}\left(s^{\prime} r^{\prime}\right) ;\right| \exp \left[\mathrm{i} \hat{\tilde{H}}\left(t^{\prime}-t\right)\right]\right. \\
& \times \mathrm{i}^{3 d(d-1) / 2} \delta^{(d)}\left(\chi^{i}\right)|\chi, K||Q(s r) ; 1, \ldots, k\rangle \\
& +\left\langle Q^{\prime}\left(s^{\prime} r^{\prime}\right) ; 1, \ldots, d \mathrm{i}^{-3 d(d-1) / 2} \delta^{(d)}\left(\chi^{\prime i}\right)\left|\chi^{\prime}, K^{\prime}\right|\right.
\end{aligned}
$$

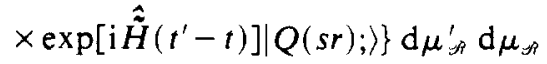

$$
\begin{aligned}
& =\frac{1}{2} \iint_{A}\left\langle Q^{\prime}\left(s^{\prime} r^{\prime}\right) \mid \exp \left[\mathrm{i} \hat{H}\left(t^{\prime}-t\right)\right] Q(s r)\right\rangle \\
& \times\left(\delta^{(d)}\left(\chi^{i}\right)|\chi, K|+\delta^{(d)}\left(\chi^{\prime i}\right)\left|\chi^{\prime}, K^{\prime}\right|\right) \mathrm{d} \mu_{\not A}^{\prime} \mathrm{d} \mu_{\star}
\end{aligned}
$$


where the inner product on the extended Hilbert space, equation (29), has been used. Note that, in arriving at the last line of (62), it is crucially important that the gauge conditions $\chi^{i}(Q)$ be independent of the momenta, $P_{\alpha}$.

Now

$$
\left\langle Q^{\prime}\left(s^{\prime} r^{\prime}\right)\left|\exp \left[\mathrm{i} \hat{H}\left(t^{\prime}-t\right)\right]\right| Q(s r)\right\rangle=\left\langle Q^{\prime}, t^{\prime} \mid Q, t\right\rangle
$$

is the amplitude to go from $\left|Q^{\prime}\right\rangle$ at time $t^{\prime}$ to $|Q\rangle$ at time $t$ on $\mathscr{S}$ and is known exactly for Lie groups $[14,15]$. Thus we arrive at the final result

$\left\langle q^{\prime}, t^{\prime} \mid q, t\right\rangle=\frac{1}{2} \iint_{\mathscr{A}}\left\langle Q^{\prime}, t^{\prime} \mid Q, t\right\rangle\left(\delta^{(d)}\left(\chi^{i}\right)|\chi, K|+\delta^{(d)}\left(\chi^{\prime i}\right)\left|\chi^{\prime}, K^{\prime}\right|\right) \mathrm{d} \mu_{: A}^{\prime} \mathrm{d} \mu_{: R}$

for the amplitude $\left\langle q^{\prime}, t^{\prime} \mid q, t\right\rangle$ for a particle to propagate from $q$ to $q^{\prime}$ on $\mathscr{S} / \mathscr{R}$ in terms of the known amplitudes $\left\langle Q^{\prime}, t^{\prime} \mid Q, t\right\rangle$ for propagation from $Q$ to $Q^{\prime}$ on $\mathscr{Y}$.

By way of demonstration, we shall now apply this machinery to an explicit example.

\section{An example: $\mathrm{SU}(2) / \mathrm{U}(1)$}

To illustrate the procedures described in the preceding sections, consider the simple case of the symmetric space $S^{2} \approx \mathrm{SU}(2) / \mathrm{U}(1)$. The results of this section are not new as the quantum mechanics of a particle moving on $S^{2}$ have been treated before (see for example $[12,16,19]$ ) but they give a simple example of the techniques developed in this paper for more general coset spaces. It will be shown that the energy eigenfunctions for a free particle moving on the 2-sphere (which are just the Legendre polynomials, $P_{J}(\cos \gamma)$ ) can be obtained from those of a free particle moving on a 3 -sphere, $\sin l \Gamma / \sin \Gamma$, by the techniques of section 4 ( $\Gamma$ is the geodesic distance between two points on $S^{3}$ ).

Since $S U(2) \approx S^{3}$ we first of all consider the geometry of $S^{3}$. This is easily described by embedding $S^{3}$ in $\mathbb{R}^{4}=\mathbb{C}^{2}$. Let $(x, y, z, t)$ be Cartesian coordinates for $\mathbb{R}^{4}$ and $(x+\mathrm{i} y, z+\mathrm{i} t)$ be complex coordinates for $\mathbb{C}^{2}$, then the Euler angles $0 \leqslant \theta \leqslant \pi, 0 \leqslant \phi \leqslant 2 \pi$ and $0 \leqslant \psi<4 \pi$ on $S^{3}$ are defined by

$$
z+\mathrm{i} t=r \sin (\theta / 2) \exp [\mathrm{i}(\psi-\phi) / 2] \quad x+\mathrm{i} y=r \cos (\theta / 2) \exp [\mathrm{i}(\psi+\phi) / 2]
$$

where $0 \leqslant r<\infty$ is a radial coordinate. The $\mathrm{SU}(2)$ symmetric metric on the $S^{3}$ of unit radius is obtained by setting $r=1$ in the Euclidian metric on $\mathbb{C}^{2}$ :

$$
\mathrm{d} s^{2}=\frac{1}{4}\left[\mathrm{~d} \theta^{2}+\cos ^{2}(\theta / 2)(\mathrm{d} \psi+\mathrm{d} \phi)^{2}+\sin ^{2}(\theta / 2)(\mathrm{d} \psi-\mathrm{d} \phi)^{2}\right] .
$$

The orthonormal Killing vectors for this metric are

$$
\begin{aligned}
& \boldsymbol{K}_{1}=2\left(\sin \psi \partial_{\theta}-\frac{\cos \psi}{\sin \theta} \partial_{\phi}+\frac{\cos \psi}{\tan \theta} \partial_{\psi}\right) \\
& \boldsymbol{K}_{2}=-2\left(\cos \psi \partial_{\theta}+\frac{\sin \psi}{\sin \theta} \partial_{\phi}-\frac{\sin \psi}{\tan \theta} \partial_{\psi}\right) \\
& \boldsymbol{K}_{3}=2 \partial_{\psi}
\end{aligned}
$$

with the usual SU(2) algebra

$$
\left[\boldsymbol{K}_{A}, \boldsymbol{K}_{B}\right]=-2 \varepsilon_{A B}{ }^{C} \boldsymbol{K}_{C} .
$$


The Laplacian can be written as

$$
\begin{aligned}
\square & =\sum_{A} \boldsymbol{K}_{A} \boldsymbol{K}_{A} \\
& =\frac{4}{\sin \theta} \partial_{\theta}\left(\sin \theta \partial_{\theta}\right)+\frac{1}{\cos ^{2}(\theta / 2)}\left(\partial_{\psi}+\partial_{\phi}\right)^{2}+\frac{1}{\sin ^{2}(\theta / 2)}\left(\partial_{\psi}-\partial_{\phi}\right)^{2} .
\end{aligned}
$$

In order to avail ourselves of the results of $[14,15]$ for the path integral on a group manifold (in this case $\mathrm{SU}(2)$ ), it is necessary to change to a coordinate system which is designed to exhibit the geodesic distance between two points. Let $\hat{n}$ be a unit vector in $\mathbb{R}^{4}=\mathbb{C}^{2}$, which represents a point on $S^{3} . \hat{n}$ has components

$\hat{\boldsymbol{n}}=\left(\cos (\theta / 2) \cos \psi_{+}, \cos (\theta / 2) \sin \psi_{+}, \sin (\theta / 2) \cos \psi_{-}, \sin (\theta / 2) \sin \psi_{-}\right)$

where $\psi_{ \pm}=\frac{1}{2}(\psi \pm \phi)$. Then the geodesic distance between two points on $S^{3}, \hat{n}$ and $\hat{n}^{\prime}$, is given by $\Gamma$ where

$\cos \Gamma=\hat{\boldsymbol{n}}^{\prime} \cdot \hat{\boldsymbol{n}}=\cos \left(\frac{\theta}{2}\right) \cos \left(\frac{\theta^{\prime}}{2}\right) \cos \left(\psi_{+}-\psi_{+}^{\prime}\right)+\sin \left(\frac{\theta}{2}\right) \sin \left(\frac{\theta^{\prime}}{2}\right) \cos \left(\psi_{-}-\psi_{-}^{\prime}\right)$.

Because of the symmetry of $S^{3}, \hat{n}$ can be taken to be the north pole, $(x, y, z, t)=$ $(1,0,0,0),(r, \theta, \phi, \psi)=(1,0,0,0)$, without loss of generality. This gives the definition of a new coordinate system, $\left(\Gamma, \psi_{+}^{\prime}, \psi_{-}^{\prime}\right)$. In these geodesic coordinates, the Laplacian takes the form

$$
\square=\partial_{\Gamma}^{2}+\frac{2}{\tan \Gamma} \partial_{\Gamma^{+}}+\frac{\tan \psi_{+}^{\prime}}{\tan \Gamma} \partial_{\Gamma^{\prime}} \partial_{\psi_{+}}+\partial_{\psi_{+}}^{2}+\frac{\cos ^{2} \psi_{+}^{\prime}}{\cos ^{2} \psi_{+}^{\prime}-\cos ^{2} \Gamma} \partial_{\psi_{-}}^{2} .
$$

Spherically symmetric eigenfunctions of this Laplacian, independent of $\psi_{+}^{\prime}$ and $\psi_{-}^{\prime}$, are given by $\sin l \Gamma / \sin \Gamma$ with

$$
\left(\partial_{\Gamma}^{2}+\frac{2}{\tan \Gamma} \partial_{\Gamma}\right)\left(\frac{\sin l \Gamma}{\sin \Gamma}\right)=-\left(l^{2}-1\right) \frac{\sin l \Gamma}{\sin \Gamma}
$$

$l=1,2,3, \ldots$ Thus the energy eigenvalues are $E_{l}=\frac{1}{2}\left(l^{2}-1\right)$.

The exact finite-time amplitude for a unit mass particle on the unit $S^{3}$ to go from the north pole at time $t$ to the point $\left(\theta^{\prime}, \phi^{\prime}, \psi^{\prime}\right)$, a geodesic distance $\Gamma$ away from the north pole at time $t^{\prime}$ is [14-16]

$$
\begin{aligned}
\left\langle\Gamma, t^{\prime} \mid 0, t\right\rangle & =\frac{1}{2 \pi^{2}} \frac{1}{\sin \Gamma} \sum_{l=1}^{\infty} l(\sin l \Gamma) \exp \left[-\mathrm{i}\left(l^{2}-1\right)\left(t-t^{\prime}\right) / 2\right] \\
& =\frac{1}{2 \pi^{2}} \frac{1}{\sin \Gamma} \sum_{j=0,1 / 2,1, \ldots}^{\infty}(2 j+1)[\sin (2 j+1) \Gamma] \exp \left[-\mathrm{i} 2 j(j+1)\left(t-t^{\prime}\right)\right]
\end{aligned}
$$

where the coordinates $\left(\Gamma, \psi_{+}^{\prime}, \psi_{-}^{\prime}\right)$ are related to $\left(\theta^{\prime}, \phi^{\prime}, \psi^{\prime}\right)$ by $\cos \Gamma=\cos \left(\theta^{\prime} / 2\right) \cos \psi_{+}^{\prime}$. The normalization is determined by demanding that, when $t=t^{\prime}$, the amplitude is a $\delta$ function, which gives unity when integrated over $S^{3} .2 \pi^{2}$ is just the volume of the unit 3-sphere.

To relate this to the exact finite-time amplitude for a particle moving on $S^{2}$ a single $(d=1)$ gauge choice is necessary. We will choose the gauge condition (in coordinates $(\theta, \phi, \psi))$

$$
\chi= \begin{cases}\psi-\phi & 0 \leqslant \psi<2 \pi \\ \psi-2 \pi-\phi & 2 \pi \leqslant \psi<4 \pi\end{cases}
$$


The U(1) orbits of $\mathscr{R}$ are generated by

$$
\boldsymbol{K}_{3}=2 \frac{\partial}{\partial \psi}=2 \mathrm{i} \hat{P}_{\psi}
$$

with Faddeev-Popov determinant $\left|\operatorname{det}\left\{\chi, K_{3}\right\}\right|=2$. Putting this gauge condition into the equation for coset decomposition of orthonormal 1 -forms (45) and using the explicit metric on $S^{3}(66)$, we see that it projects the three-dimensional sphere of unit radius to a two-dimensional sphere of radius $\frac{1}{2}$. This is just the standard Hopf fibration of the 3-sphere. With this gauge choice, the measure on $\mathrm{U}(1) \approx \mathscr{R}$ is just $\mathrm{d} \psi / 2$.

The relative normalizations of the metrics on $S^{2}$ and $U(1)$ can also be obtained from the analysis of [7] as described in section 3. Using the definition of the measure on $\tilde{\mathscr{H}}_{f}$ (equations (23) and (24)) with

$$
K_{3}=2 P_{\psi}
$$

and $G^{A B}$ given by the inverse metric of (66)

$$
G^{\alpha \beta}=4\left(\begin{array}{ccc}
1 & 0 & 0 \\
0 & 1 / \sin \theta & -\cos \theta / \sin ^{2} \theta \\
0 & -\cos \theta / \sin ^{2} \theta & 1 / \sin \theta
\end{array}\right)
$$

we find

$$
\|K\|=\frac{1}{8} \sin \theta=\sqrt{G} .
$$

Thus, if the measure on $S^{2}$ is $\mathrm{d} \mu_{\mathscr{S} / \mathscr{R}}=\frac{1}{4} \sin \theta \mathrm{d} \theta \mathrm{d} \phi$, then the measure on $\mathscr{R}$ is $\mathrm{d} \mu_{\mathscr{R}}=$ $\mathrm{d} \psi / 2$.

Note that the two cases of the function $\chi$ in equation (75) are necessary in order to project out the whole of $S^{2}$ from $S^{3}$. The range $0 \leqslant \psi<2 \pi$ would only give one hemisphere of $S^{2}$, as an examination of (65) with $r=1$ shows. This is an inevitable consequence of the fact that $S^{3}$ is a non-trivial $U(1)$ bundle over $S^{2}$ and means that $\chi$ has two zeros on each gauge orbit instead of one. Because of this an extra factor of a half is required in the normalization of the states, as described below.

Let $q$ be the point on $S^{2}$ corresponding to the coset of SU(2) containing the point $(\theta, \phi, \psi)$ of $S^{3}$ and $q^{\prime}$ be the point on $S^{2}$ corresponding to the coset containing the point $\left(\theta^{\prime}, \phi^{\prime}, \psi^{\prime}\right)$ of $S^{3}$. Then, by the analysis of section 4 and equation (64),

$$
\begin{aligned}
\left\langle q^{\prime}, t^{\prime} \mid q, t\right\rangle & ={ }_{\chi}\left\langle q^{\prime}, t^{\prime} ; \mid q, t ;\right\rangle_{\chi} \\
& =\frac{1}{8} \int_{0}^{4 \pi} \int_{0}^{4 \pi}\left\langle\theta^{\prime}, \phi^{\prime}, \psi^{\prime}\left|\exp \left[\mathrm{i} \hat{H}\left(t^{\prime}-t\right)\right]\right| \theta, \phi, \psi\right\rangle\left(\delta\left(\chi^{\prime}\right)+\delta(\chi)\right) \mathrm{d} \psi \mathrm{d} \psi^{\prime}
\end{aligned}
$$

where an extra factor of a half has been inserted to allow for the fact that the gauge fixing function, $\chi$ in equation (75), has two zeros on each gauge orbit. Using the exact expression (74), we have

$$
\left\langle q^{\prime}, t^{\prime} \mid q, t\right\rangle=\frac{1}{16 \pi^{2}} \int_{0}^{4 \pi} \mathrm{d} \psi \int_{0}^{4 \pi} \mathrm{d} \psi^{\prime} \sum_{l=1}^{x} \frac{l \sin l \Gamma}{\sin \Gamma} \exp \left[-\mathrm{i}\left(l^{2}-1\right)\left(t-t^{\prime}\right) / 2\right]\left(\delta\left(\chi^{\prime}\right)+\delta(\chi)\right)
$$

where $\Gamma$ is a function of $\theta^{\prime}, \phi^{\prime}, \psi^{\prime}$ and $\theta, \phi, \psi$. To evaluate this expression we need to 
perform the integrals

$$
\begin{aligned}
I_{i}=\int_{0}^{4 \pi} \mathrm{d} \psi & \int_{0}^{4 \pi} \mathrm{d} \psi^{\prime} \frac{\sin l \Gamma}{\sin \Gamma}\left(\delta\left(\chi^{\prime}\right)+\delta(\chi)\right) \\
= & \int_{0}^{4 \pi} \mathrm{d} \psi \int_{0}^{2 \pi} \mathrm{d} \psi^{\prime} \frac{\sin l \Gamma}{\sin \Gamma} \delta\left(\psi^{\prime}-\phi^{\prime}\right) \\
& +\int_{0}^{4 \pi} \mathrm{d} \psi \int_{2 \pi}^{4 \pi} \mathrm{d} \psi^{\prime} \frac{\sin l \Gamma}{\sin \Gamma} \delta\left(\psi^{\prime}-2 \pi-\phi^{\prime}\right) \\
& + \text { two similar terms with }\left(\phi^{\prime}, \psi^{\prime}\right) \leftrightarrow(\phi, \psi) .
\end{aligned}
$$

To do this we need to express $\Gamma$ in terms of $(\theta, \phi, \psi)$ and $\left(\theta^{\prime}, \phi^{\prime}, \psi^{\prime}\right)$ using its definition (71). We can take $\theta=0$ and $\phi=0$ without loss of generality, so that

$$
\cos \Gamma=\cos \left(\theta^{\prime} / 2\right) \cos \left(\frac{\psi-\psi^{\prime}-\phi^{\prime}}{2}\right) \text {. }
$$

Using the expansion

$\frac{\sin l \Gamma}{\sin \Gamma}=(2 \cos \Gamma)^{l-1}-\left(\begin{array}{c}l-2 \\ 1\end{array}\right)(2 \cos \Gamma)^{l-3}+\left(\begin{array}{c}l-3 \\ 2\end{array}\right)(2 \cos \Gamma)^{l-5}-\ldots$

and

$$
\int_{0}^{2 \pi}(\cos \lambda)^{l} \mathrm{~d} \lambda= \begin{cases}2 \pi \frac{(l-1)(l-3) \ldots 5.3 .1}{l(l-2) \ldots 2} & \text { if } l \text { is even } \\ 0 & \text { if } l \text { is odd }\end{cases}
$$

we find that all four terms in $I_{l}$ are identical and

$I_{l}= \begin{cases}16 \pi \sum_{p=0}^{j}(-1)^{p}\left(2 \cos ^{2}\left(\theta^{\prime} / 2\right)\right)^{j-p} \frac{(2 j-p) !}{2^{j-p} p ![(j-p) !]^{2}} & \text { if } l \text { is even } \\ 0, & \text { if } l \text { is odd }\end{cases}$

where we have set $j=(l-1) / 2$.

It is now a trivial matter to prove, e.g. using Rodrigues' formula

$$
P_{j}\left(\cos \theta^{\prime}\right)=\frac{1}{2^{j} j !} \frac{\mathrm{d}^{\prime}}{\mathrm{d}\left(\cos \theta^{\prime}\right)^{j}}\left(\cos ^{2} \theta^{\prime}-1\right)^{j}
$$

that

$$
I_{l}=16 \pi P_{j}\left(\cos \theta^{\prime}\right)
$$

Since $S^{2}$ is homogeneous we can treat the more general case, when $\hat{n}$ does not necessarily have $\theta=\phi=0$ by replacing $\theta^{\prime}$ with the geodesic distance, $\gamma$, between $q^{\prime}$ and $q$ on $S^{2}$.

Thus the equation for the propagator of a free particle on a two-dimensional sphere of radius $\frac{1}{2}$ in terms of an integral over the extended Hilbert space (64) gives

$$
\left\langle q^{\prime}, t^{\prime} \mid q, t\right\rangle=\frac{1}{\pi} \sum_{j=0}^{x}(2 j+1) P_{j}(\cos \gamma) \exp \left[-\mathrm{i} 2 j(j+1)\left(t-t^{\prime}\right)\right] .
$$

More generally, for a 2 -sphere of radius $R$, the result is

$$
\left\langle q^{\prime}, t^{\prime} \mid q, t\right\rangle=\frac{1}{4 \pi R^{2}} \sum_{j=0}^{x}(2 j+1) P_{j}(\cos \gamma) \exp \left(-\mathrm{i} \frac{j(j+1)\left(t-t^{\prime}\right)}{2 R^{2}}\right)
$$

in full agreement with $[12,16]$. 


\section{Conclusions}

It has been shown how the use of ghosts enables the quantum mechanics of a free, non-relativistic particle moving on a homogeneous space $\mathscr{S} / \mathscr{R}$ to be treated in a consistent manner, by considering it to be a reduced system coming from a free particle moving on the Lie group $\mathscr{F}$ where the problem has already been solved. In particular, the results of $[7,8]$ ensure that the procedures of quantizing first and then reducing or reducing first and then quantizing actually commute.

The propagator on $\mathscr{F} / \mathscr{R}$ is obtained by first extending the Hilbert space for the problem on a Lie group to a pseudo-Hilbert space, imposing gauge conditions in a BRST-invariant manner and then integrating out the redundant degrees of freedom. The finite-time propagator on $\mathscr{F}$ is already known exactly $[14,15]$ and this allows the finite-time propagator on $\mathscr{S} / \mathscr{R}$ to be evaluated in a gauge invariant manner, leading to equation (64) for the amplitude for a particle to propagate from $q$ to $q^{\prime}$ on $\mathscr{S} / \mathscr{R}$ in terms of the known amplitude to propagate from $Q$ to $Q^{\prime}$ on $\mathscr{S}$.

The example of $S^{2} \approx \mathrm{SU}(2) / \mathrm{U}(1)$ was treated explicitly and it was shown how the Legendre polynomials for the eigenvalue problem on $S^{2}$ could be obtained from the eigenfunctions $\sin l \Gamma / \sin \Gamma$ on $S^{3}$, by choosing a gauge and integrating. There is a subtlety in the gauge choice in that, since $S^{3}$ is a non-trivial U(1) bundle over $S^{2}$, the gauge condition $\chi$ had more than one zero on each gauge orbit and it was necessary to divide the amplitude by the number of zeros in order to ensure the correct normalization. One can expect this to be a generic feature of this procedure for calculating amplitudes on homogeneous spaces.

\section{References}

[1] Feynman R P 1963 Acta Phys. Pol. 26697

[2] Faddeev L D and Popov V N 1967 Phys. Lett. 25B 29

[3] Hennaux M and Teitelboim C 1988 Commun. Math. Phys. 115213

[4] Hennaux M 1985 Phys. Rep. 1261

[5] Bonora L and Cotta-Ramusino P 1983 Commun. Math. Phys. 87589

[6] Dirac P A M 1987 Lectures on Quantum Mechanics (New York: Academic)

[7] McMullan D and Paterson J 1989 J. Math. Phys. 30477

[8] McMullan D and Paterson J 1989 J. Math. Phys. 30487

[9] Dowker J S 1972 J. Phys. A: Math. Gen. 5936

[10] Dowker J S 1972 Int. J. Theor. Phys. 8319

[11] Benabdallah A 1973 Bull. Soc. Math. France 101265

[12] Böhm M and Junker G 1988 Group theoretical approach to path integration on spheres Paih Summation: Achievements and Goals (Proc. Adriatico Research Conf. on Path Integral Methods With Applications, 1987 Trieste) ed S Lundvist, A Ranfagni, V Sa-yakanit and L S Schulman (Singapore: World Scientific)

[13] Anderson A and Camporesi R 1989 Intertwinning operators for solving differential equations with applications to symmetric spaces Preprint University of Utah

[14] Dowker J S 1970 J. Phys. A: Math. Gen. 3451

[15] Dowker J S 1970 Ann. Phys. 62361

[16] Schulmann L 1981 Techniques and Applications of Path Integration (New York: Wiley)

[17] Gribov V N 1978 Nucl. Phys. B 1391

[18] Kobayashi S and Nomizu K 1969 Foundations of Differential Geometry: Vol. II (New York: Wiley)

[19] Anderson A and Anderson S B 1989 Phase space path integration of integrable quantum systems Preprint University of Utah 